\title{
Evaluation of Elite Open-Pollinated Maize Lines in Two Contrasting Environments
}

\author{
Vasileios Greveniotis ${ }^{1,2}$, Elisavet Bouloumpasi ${ }^{3}$, Ioannis Tsakiris ${ }^{3}$, Evangelia Sioki ${ }^{4} \&$ Constantinos Ipsilandis $^{5}$ \\ ${ }^{1}$ Department of Agricultural Technology, Technological Education Institute of Thessaly, Larissa, Greece \\ ${ }^{2}$ Department of Agricultural Development, Democritus University of Thrace, Orestiada, Greece \\ ${ }^{3}$ Department of Agricultural Technologists, School of Agricultural Technology, Food Technology and Nutrition, \\ Technological Educational Institution of Western Macedonia, Florina, Greece \\ ${ }^{4}$ National Center for Quality Control, Classification \& Standardization of Cotton, Hellenic Agricultural \\ Organization-"Demeter", Karditsa, Greece \\ ${ }^{5}$ Department of Agriculture, Regional Administration of Central Macedonia, Thessaloniki, Greece \\ Correspondence: Vasileios Greveniotis, Department of Agricultural Technology, Technological Education \\ Institute of Thessaly, Larissa, Greece. E-mail: vgreveni@mail.com
}

Received: October 24, 2017

doi:10.5539/jas.v10n1p 85
Accepted: November 27, $2017 \quad$ Online Published: December 15, 2017

URL: https://doi.org/10.5539/jas.v10n1p85

\begin{abstract}
Selection environment affects plant behavior and response to selection. The objective of the present study was the evaluation of 17 quality and quantity phenotypic characteristics of six open-pollinated maize lines of fifth cycle of selection (C4), which was performed by the implementation of honeycomb breeding, in two contrasting environments (A and B). A: Florina, W. Macedonia $\left(40^{\circ} 46^{\prime} \mathrm{N}, 21^{\circ} 22^{\prime} \mathrm{E}\right.$, altitude $\left.705 \mathrm{~m}\right)$ and B: Trikala, Thessaly $\left(39^{\circ} 55^{\prime} \mathrm{N}, 21^{\circ} 64^{\prime} \mathrm{E}\right.$, altitude $\left.120 \mathrm{~m}\right)$, with about $4-10{ }^{\circ} \mathrm{C}$ higher temperatures than environment $\mathrm{A}$. The soil chemical analysis revealed that the two environments were very diverse (A: $\mathrm{SL}, \mathrm{pH}=6.25$, organic matter: $1.29 \%$, B: SCL, $\mathrm{pH}=8$, organic matter: $2.4 \%$ ). Our data suggest that moisture content, seed oil content, ear length, ear diameter, number of grain rows, spindle diameter and seed thickness exhibit inter-location high broad-sense heritability (over 0.9 ). Heritability estimations were highly depended on the environment, since GEI interaction was high indicating environmental interaction with genotype, especially environment $B$, which seems to favor heritability. Location affects strongly variation and genotype by environment interaction is significant in many cases. Seed width was the only characteristic to be depended on genetic variability. Descriptive statistics revealed a broad range of mean fluctuations, indicating satisfactory variability in many characteristics to be exploited by breeders. Some characteristics showed low CV (Coefficient of Variation) values (1.6 to 5.3), indicating stability of performance and low environmental effects. Significant correlations between the 17 quantity and quality traits found in our study may be a useful tool for indirect selection of certain characteristics, otherwise difficult to be selected due to non-additive effects. Cluster analysis and PCA showed contrasting results in classification of open-pollinated lines and this was attributed to strong environmental effects that distorted phenotypic expression of the characteristics studied.
\end{abstract}

Keywords: color analysis, ear traits, honeycomb design, physicochemical analysis

\section{Introduction}

Maize (Zea mays L.) is one of the most important cereal crops and the second in quantity agricultural product after sugar cane. It is cultivated for human consumption, as well as feed (Nuss \& Tanumihardjo, 2010). Moreover, it is used as raw material for beer production and alcoholic beverage production after mashing, fermentation and distillation (Russell \& Stewart, 2014). It may also be further processed in order to produce food additives (Nuss \& Tanumihardjo, 2010). As a result of its wide use and its contribution to global nutrition, the composition of maize is of high interest. Maize kernel is constituted by starch, protein, oil, fiber, vitamins and nutrients (L. Wang, Q. Wang, H. Liu, L. Liu \& Du, 2013). However, composition of maize is affected by several factors, including environmental conditions, parenting of the maternal plant and genetics of the endosperm sink (Nuss \& Tanumihardjo, 2010). 
The measurement of maize's quality variables employing Near-Infrared (NIR) reflectance spectroscopy is rapid and accurate. In maize compositional analysis, NIR spectroscopy has been only used to analyze protein and amino acids (Rosales, Galicia, Oviedo, Islas, \& Palacios-Rojas, 2011), as well as kernel density and volume (Gustin et al., 2013). The determination of physicochemical and biochemical characteristics is significant for the breeding and selection processes (Motto, Hartings, Fracassetti, \& Consonni, 2011; Rosales, Galicia, Oviedo, Islas, \& Palacios-Rojas, 2011). Although maize breeding succeeded in increasing field yield, quality has received less attention even though maize breeders achieved in developing kernels with various characteristics and composition (Motto, Hartings, Fracassetti, \& Consonni, 2011). Differences in physical and biochemical kernel traits among modern maize hybrids tend to be much smaller than in old landraces and open-pollinated varieties (Seebauer et al., 2010). Quality of kernel is more enriched in modern maize varieties as a result of intensive breeding but in general, genetic diversity is small and genetic erosion seems to be a threat (Jaradat \& Goldstein, 2013).

Multivariate analysis is preferred for analyzing data derived from both quality and quantity characteristics (de Oliveira, Dias, \& Dantas, 2012). Multivariate techniques such as Principal Component Analysis (PCA) and Agglomerative Hierarchical Clustering (AHC) analysis have been previously used for morphological assessment of genetic variability in sweet cherry (Prunus avium L.) cultivars (Ganopoulos et al., 2015) and in maize (Alves, Cargnelutti Filho, Toebe, Burin, \& Silva, 2014).

Honeycomb breeding and designs, maximize phenotypic expression and differentiation under nil-competition conditions, weakening also soil heterogeneity effects, and thus, resulting in selection of superior individual plants (Fasoulas \& Fasoula, 1995; Fasoulas, 1988). This way, plants are enabled to express their full genetic potential for tillering, yielding capacity, robust root systems and full phenotypic expression.

The objective of the present study was the evaluation of diverse phenotypic characteristics of six open-pollinated maize lines of fifth cycle of selection (C4), which was performed by the implementation of honeycomb breeding as a means of maximizing phenotypic expression, in two contrasting environments (different regions).

\section{Materials and Methods}

\subsection{Plant Materials}

The starting material was the F2 (C0) generation of the F1 commercial maize hybrid Costanza. Selection was performed in honeycomb selection designs, which allow the individual plants to express their full genetic potential (Fasoulas \& Fasoula, 1995) for five cycles. A total of six high-yielding open-pollinated lines of the $5^{\text {th }}$ cycle (C4) have been selected on the basis of the two prognostic equations (Fasoula, 2006) with selection intensity near $1 \%$. Despite the use of open-pollination, the pedigree selection scheme was conducted for all years of experimentation on the half-sib progenies. In 2012, the six open-pollinated lines along with the F1 maize hybrid Costanza (Table 1) were sown in a R7 honeycomb design (Fasoulas \& Fasoula, 1995) in two contrasting environments A: Florina, W. Macedonia $\left(40^{\circ} 46^{\prime} \mathrm{N}, 21^{\circ} 22^{\prime} \mathrm{E}\right.$, altitude $\left.705 \mathrm{~m}\right)$ and B: Trikala, Thessaly $\left(39^{\circ} 55^{\prime} \mathrm{N}\right.$, $21^{\circ} 64^{\prime} \mathrm{E}$, altitude $120 \mathrm{~m}$, with about $4-10^{\circ} \mathrm{C}$ higher temperatures than environment A). The soil chemical analysis revealed that the two environments were very diverse (A: SL-Silica Loam, $\mathrm{pH}=6.25$, organic matter: $1.29 \%$, B: SCL-Sand Silica Loam, $\mathrm{pH}=8$, organic matter: $2.4 \%$ ). Mean, High and Low monthly temperatures in the two environments are presented in Figure 1. 
Table 1. The genetic materials used in experiments in the two environments

\begin{tabular}{llll}
\hline & Location & Honeycomb family & Genetic material \\
\hline 1 & Environment B & Trikala 1 & Open-pollinated-1 \\
2 & Environment B & Trikala 2 & Open-pollinated-2 \\
3 & Environment B & Trikala 3 & Open-pollinated-3 \\
4 & Environment B & Trikala 4 & Open-pollinated-4 \\
5 & Environment B & Trikala 5 & Open-pollinated-5 \\
6 & Environment B & Trikala 6 & Open-pollinated-6 \\
7 & Environment B & Trikala 7 & F Costanza \\
8 & Environment A & Florina 1 & Open-pollinated-1 \\
9 & Environment A & Florina 2 & Open-pollinated-2 \\
10 & Environment A & Florina 3 & Open-pollinated-3 \\
11 & Environment A & Florina 4 & Open-pollinated-4 \\
12 & Environment A & Florina 5 & Open-pollinated-5 \\
13 & Environment A & Florina 6 & Open-pollinated-6 \\
14 & Environment A & Florina 7 & F Costanza \\
\hline
\end{tabular}
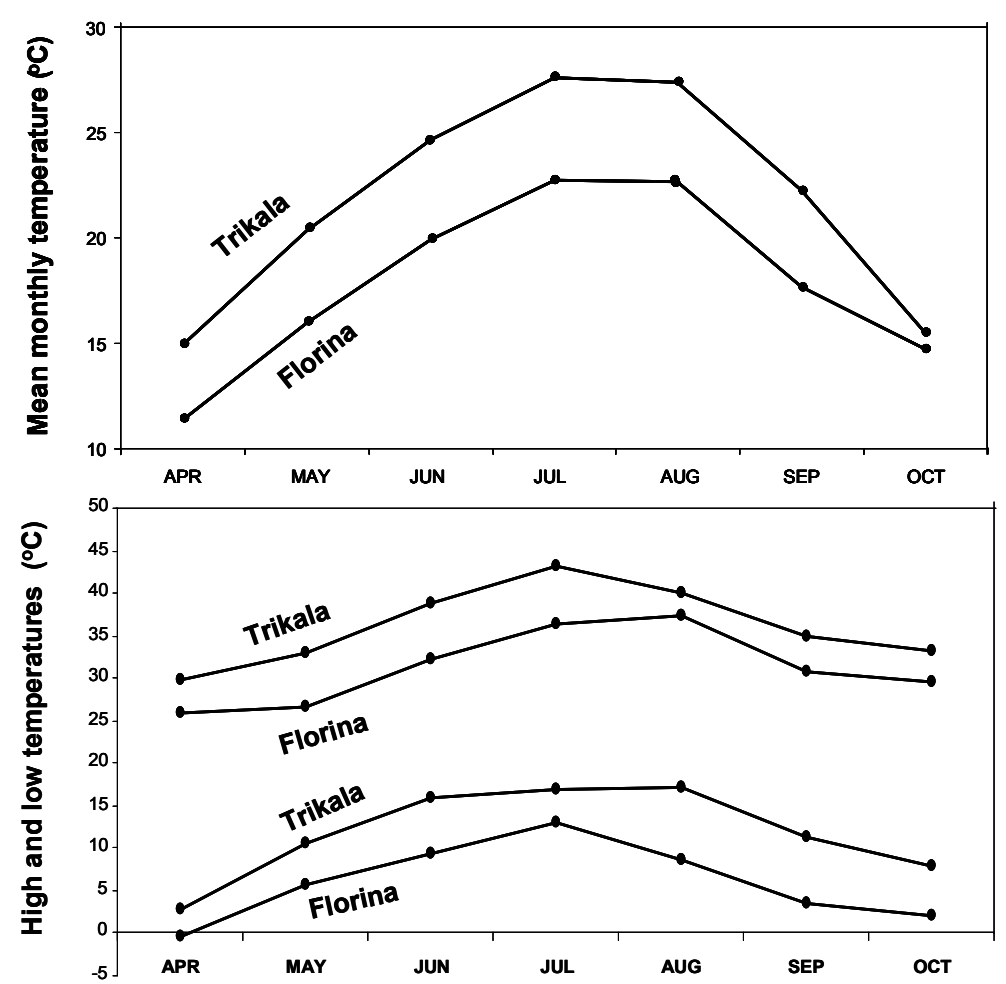

Figure 1. Mean, High and Low monthly temperatures in the two contrasting environments (Trikala and Florina), from April to October

Nitrogen and phosphorus fertilizer were applied (since it is well known by Hellenic Agricultural Organization"Demeter" that these fields have abundance of potassium: $260 \mathrm{mg} / \mathrm{kg}$ ) at the rate of 150 and $75 \mathrm{~kg} \mathrm{ha}^{-1}$ respectively, at sowing, while additional $\mathrm{N}\left(135 \mathrm{~kg} \mathrm{ha}^{-1}\right)$ was applied when the plants reached $50 \mathrm{~cm}$ in height (boot stage). Weed control was obtained by tilling and manual hoeing. Evaluation and selection of individual plants were performed under ultra-wide plant spacing because competition interferes with the equal sharing of growth resources (Fasoulas, 1993). All trials were regularly irrigated after planting to avoid drought stress.

\subsection{Basics of Methodology}

Selection of individual plants was based on honeycomb methodology (Fasoulas, 1988; Fasoula, 2013). In initial populations non-replicated honeycomb designs (NR-0) were used (Figure 2), as described by Fasoulas (1988). In 
half-sib progenies, replicated designs were used as the R-7 presented in Figure 3 (Fasoulas, 1988). In all experiments, the plant-to-plant spacing was $1.25 \mathrm{~m}$ (wide-spaced, Fasoulas, 1988) and plants were grown in isolation and under open pollination. Three seeds were planted per hill and plants thinned to one plant per hill four weeks later (after planting). In non-replicated designs, superior plants and lines were selected using the plant yielding index PYI $=\left(\mathrm{X} / \overline{\mathrm{X}}_{\mathrm{r}}\right)^{2}$ proposed by Fasoula $(2006,2013)$. In replicated designs, superior plants and lines were selected on the basis of the two selection equations, the plant prognostic equation $\left(\mathrm{X} / \overline{\mathrm{X}}_{\mathrm{r}}\right)^{2} \cdot(\overline{\mathrm{X}} / \mathrm{s})^{2}$ and the line prognostic equation $\left(\overline{\mathrm{X}} / \overline{\mathrm{X}}_{\mathrm{t}}\right)^{2} \cdot(\overline{\mathrm{X}} / \mathrm{s})^{2}$, where $\mathrm{x}$ is the yield per plant, $\overline{\mathrm{X}}_{\mathrm{r}}$ is the mean plant yield of the surrounding plants within the moving ring, $\overline{\mathrm{X}}_{\mathrm{t}}$ is the overall mean plant yield of the experiment, $\overline{\mathrm{X}}$ and $\mathrm{s}$ are the entry (family) mean yield and standard deviation (Fasoula, 2006, 2013).

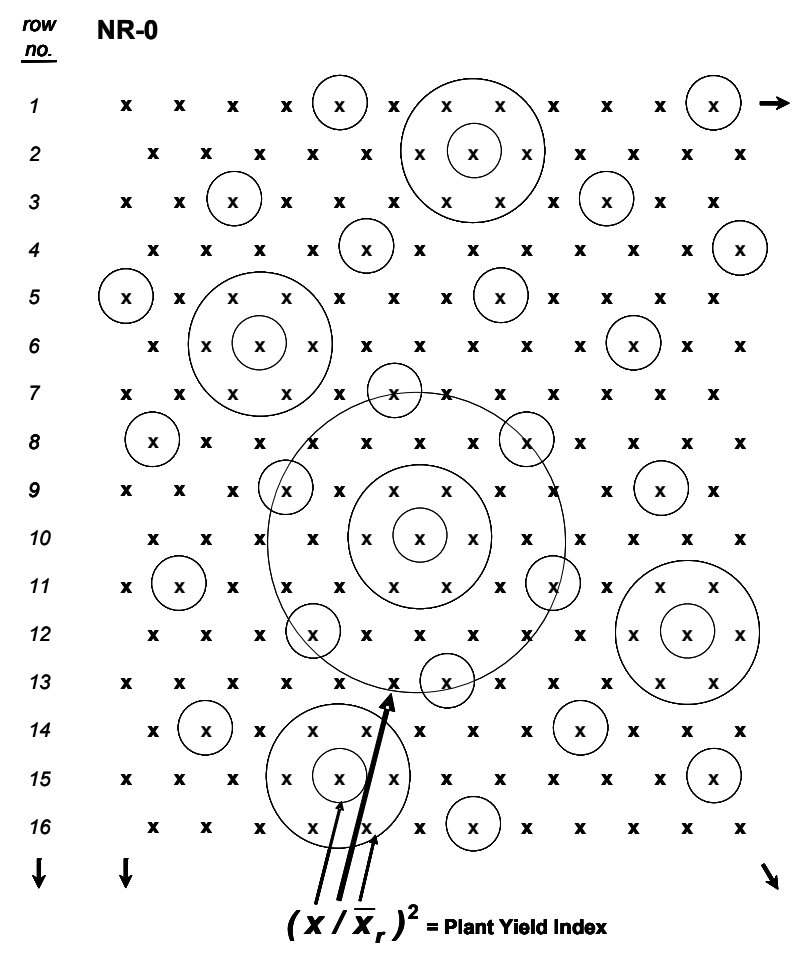

Figure 2. The honeycomb NR-0 arrangement, with selection rings and plant yielding index as selection formula (where, $\mathrm{x}$ is the yield per plant, $\overline{\mathrm{X}}_{\mathrm{r}}$ is the mean plant yield of the surrounding plants within the moving ring) 


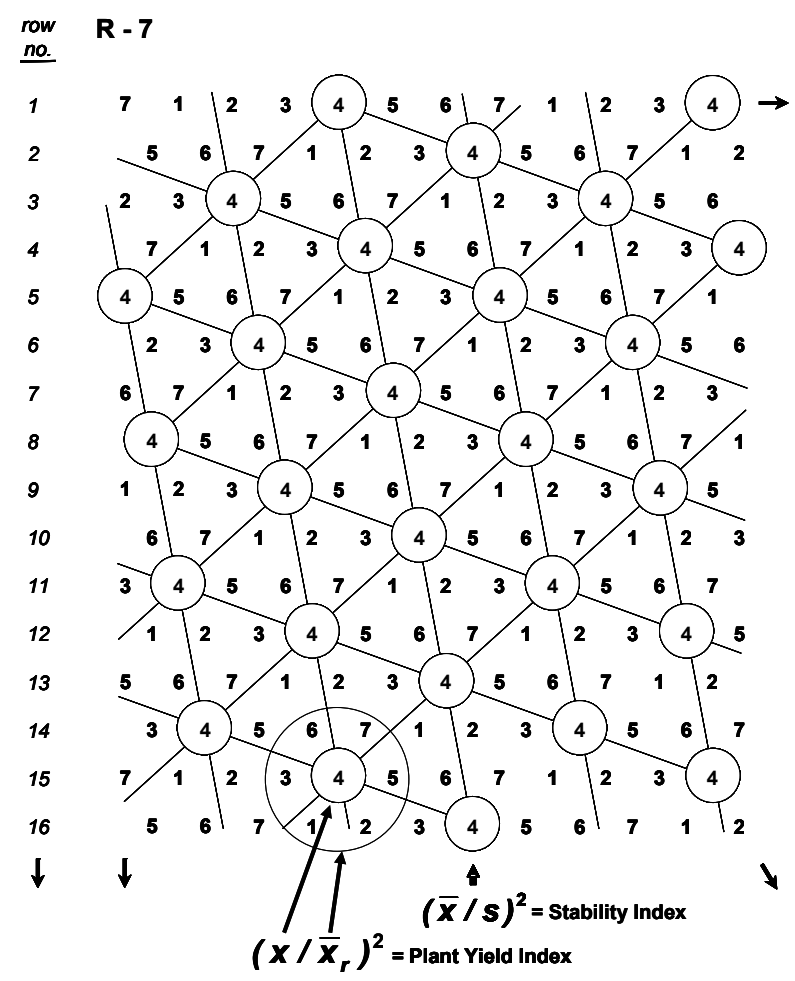

Figure 3. The replicated honeycomb R-7 arrangement, with seven entries, plant yielding index and stability index as selection formula (where, $\mathrm{X}$ is the yield per plant, $\overline{\mathrm{X}}_{\mathrm{r}}$ is the mean plant yield of the surrounding plants within the moving ring, $\overline{\mathrm{X}}$ and s are the entry (family) mean yield and standard deviation)

\subsection{Measurements and Analyses of Morphological Traits}

Measurements of quantity and quality characteristics were performed in Division of Agricultural Products Quality Control laboratory.

\subsubsection{Quality Characteristics}

The quality characteristics of maize open-pollinated lines (plus the original F1 check) were measured using SpectraStar, a Near-Infrared (NIR) reflectance spectroscopy instrument with pre-dispersive scanning monochromator that covers the range between $1200-2400 \mathrm{~nm}$. The characteristics measured were seed protein $(\%)$, seed oil $(\%)$, moisture $(\%), \mathrm{pH}$ value, ash content $(\%)$ and color characteristics $\left(\mathrm{L}^{*}, \mathrm{a}^{*}, \mathrm{~b}^{*}\right.$ parameters, corresponding to luminosity, redness and yellowness, respectively). Prior to the analyses the kernel samples have been milled.

\subsubsection{Quantity Characteristics}

The quantity characteristics measured were kernel size and weight, the number of grain rows per ear, the length and diameter of ear, the diameter of spindle. For the calculation of thousand-kernel weight (TKW), 1000 maize grains were counted and weighed. Also, the hectoliter weight was calculated as the weight of kernels per 100 litres. Kernel size was calculated in $\mathrm{mm}$ as a mean after randomly selecting 10 kernels and measuring the three major axes, namely: length, width and thickness in $\mathrm{mm}$ with a Vernier Calliper. The dimensions of ear length (in $\mathrm{cm})$, ear diameter $(\mathrm{mm})$ and spindle diameter $(\mathrm{mm})$ were measured using a ruler and a calliper and calculated as a mean of 10 ear observation for each genetic material. The number of grain rows per ear was counted by eye, as a mean of 10 ear observation for each genetic material.

\subsubsection{Data Analyses}

Analyses of the honeycomb data were based on two-way comparisons, between families and within families (Steel \& Torrie, 1980). Separation of means was based on Duncan's test. Inter-location total Sum of Squares (SS) was used to estimate the contribution of the two factors ( $\mathrm{G}$ for genetic materials and $\mathrm{E}$ for location-environment, while GEI is the interaction proportion of total SS) based on expected mean squares of the model and also, for 
each location, genotype phenotypic variance $\left(\sigma_{\mathrm{p}}^{2}\right)$ and genotype genetic variance $\left(\sigma_{\mathrm{g}}^{2}\right)$ have been calculated (McIntosh, 1983). Finally, genotypic variability coefficient (GCV), phenotypic variability coefficient (PCV), broad sense heritability $(\mathrm{H})$, expected genetic advance $(\mathrm{GA})$ and genetic advance as percentage of mean (GA\% mean) have been calculated according to Johnson, Robinson, and Comstock (1955).

Principal Components Analysis (PCA) was used to determine the number of main factors that could be used in order to decrease the necessary numbers of effective parameters for the discrimination of genotypes. Moreover, this method could allow the correlation of phenotypic traits with genetic linkage between loci controlling traits. Cluster analysis was used in order to separate the available data into groups of increasing dissimilarity. The Euclidean distance was used as a metric to measure the genetic dissimilarity of the 14 genetic materials (seven materials for two locations), based on the combined quantity and quality data and Ward's method was used for the agglomeration. Correlations were based on the procedure described by Steel and Torrie (1980) involving all quantity and quality characteristics.

\section{Results}

Statistics and variation estimations are summarized in Tables 2 and 3. Table 2, presents means' comparisons for the 13 parametric characteristics (seed protein, seed oil, moisture, ash content, kernel size and weight, number of grain rows per ear, length and diameter of ear, the diameter of spindle, thousand-kernel weight (TKW) and the hectoliter weight). For moisture (\%) content, most of the genetic materials showed high values (usually over $12 \%$ ), as well as the original hybrid Costanza, especially in environment A. Open-pollinated line five showed one of the lowest moisture (near 11.5\%) contents (inter-location data) in both environments, with statistically significant differences. Costanza and open-pollinated lines one and three showed the highest seed oil contents (exceeding 3\% inter-location). For seed protein, open-pollinated lines 5 and 6 showed the greatest values (near $10 \%$ ) with statistically significant differences. Ash content data ranged from 1.74 to 1.96 (inter-location measurements). For ear length open-pollinated line 4 showed the greatest value in environment A $(24.22 \mathrm{~cm})$, but the lowest in environment B $(18.87 \mathrm{~cm})$. Open-pollinated line five showed the greatest ear diameter, in most cases over $53 \mathrm{~mm}$. Number of grain rows was generally high and over 16, especially for open-pollinated line 3 . With regard to spindle diameter, open-pollinated line 5 showed one of the greatest values in both environments, while open-pollinated line one exceeded $31 \mathrm{~mm}$ in environment A with statistically significant differences. For seed length, Costanza, showed one of the greatest values (12.04 mm inter-location). As for seed width, environment A generally showed no statistically significant differences but inter-location values ranged from 8.58 to $9.43 \mathrm{~mm}$ with statistically significant differences. Regarding seed thickness, values ranged from 4.78 to $6.54 \mathrm{~mm}$ for environment $\mathrm{B}$, while for environment $\mathrm{A}$ the range was lower and inter-location range was even lower, even though there were statistically significant differences. Hectoliter weight was favored by environment $\mathrm{B}$, where values were over $73 \mathrm{~kg} \mathrm{~L}^{-1}$, reaching $77.32 \mathrm{~kg} \mathrm{~L}^{-1}$ for open-pollinated line one, with statistically significant differences. Thousand-kernel weight was favored by environment B, where open-pollinated line 4 was weighed and calculated at $401.5 \mathrm{~g}$, while Costanza showed the greatest values for environment $\mathrm{A}$ and inter-location (326.7 and $347.5 \mathrm{~g}$, respectively).

Table 3 presents the rest of statistics calculations: Sum of Squares ( $G$ for genetic materials and $E$ for location-environment, while GEI is the interaction proportion of total SS) and also, for each location, cultivar phenotypic variance $\left(\sigma_{\mathrm{p}}^{2}\right)$ and cultivar genetic variance $\left(\sigma_{\mathrm{g}}^{2}\right)$, as well as, genotypic variability coefficient (GCV), phenotypic variability coefficient $(\mathrm{PCV})$, repeatability $\left(\mathrm{R}^{2}\right)$, broad sense heritability $\left(\mathrm{H}^{2}\right)$, expected genetic advance (GA) and genetic advance as percentage of mean (GA\%). In general, most of the characteristics showed high $\sigma_{\mathrm{g}}^{2}$ values (values varied between characteristics) in comparison to $\sigma_{\mathrm{p}}^{2}$, resulting in high heritability that reached 0.98 for ear length in environment A, 0.95 for spindle diameter, 0.94 for ear diameter and moisture content, 0.93 for number of grain rows and seed oil content, 0.91 for seed protein. Seed thickness showed the lowest heritability value in environment A, only 0.62 . 
Table 2. Mean comparisons for each of the 13 characteristics (Moisture, Seed oil, Ash content, Seed protein, Ear length, Ear diameter, Number of grain rows per ear, Spindle diameter, Seed length, width and thickness, Hectoliter weight, Thousand kernel weight), for the two environments used (A and B) and across environments (inter-location)

\begin{tabular}{|c|c|c|c|c|c|c|c|c|c|c|c|c|c|}
\hline & Moisture & $\begin{array}{l}\text { Seed } \\
\text { oil }\end{array}$ & $\begin{array}{l}\text { Ash } \\
\text { content }\end{array}$ & $\begin{array}{l}\text { Seed } \\
\text { protein }\end{array}$ & $\begin{array}{l}\text { Ear } \\
\text { length }\end{array}$ & $\begin{array}{l}\text { Ear } \\
\text { diameter }\end{array}$ & $\begin{array}{l}\text { Number of } \\
\text { grain rows } \\
\text { per ear }\end{array}$ & $\begin{array}{l}\text { Spindle } \\
\text { diameter }\end{array}$ & $\begin{array}{l}\text { Seed } \\
\text { length }\end{array}$ & $\begin{array}{l}\text { Seed } \\
\text { width }\end{array}$ & $\begin{array}{l}\text { Seed } \\
\text { thickness }\end{array}$ & $\begin{array}{l}\text { Hectoliter } \\
\text { weight }\end{array}$ & $\begin{array}{l}\text { Thousand- } \\
\text { kernel } \\
\text { weight }\end{array}$ \\
\hline \multicolumn{14}{|l|}{ Inter-location } \\
\hline Open-pollinated-1 & $12.18 \mathrm{ab}$ & $3.06 \mathrm{a}$ & $1.81 \mathrm{bc}$ & $9.18 \mathrm{~b}$ & $19.98 \mathrm{c}$ & $47.80 \mathrm{c}$ & $15.92 \mathrm{c}$ & $27.56 \mathrm{~d}$ & $11.75 \mathrm{ab}$ & 8.79 de & $5.19 \mathrm{~cd}$ & $72.23 \mathrm{a}$ & $319.7 \mathrm{c}$ \\
\hline Open-pollinated-2 & $11.85 \mathrm{de}$ & $2.89 \mathrm{bc}$ & $1.96 \mathrm{a}$ & $9.11 \mathrm{~b}$ & $21.56 \mathrm{a}$ & $50.27 \mathrm{~b}$ & $16.79 \mathrm{~b}$ & $27.13 \mathrm{~d}$ & $11.37 \mathrm{c}$ & $9.06 \mathrm{bc}$ & $5.93 \mathrm{a}$ & $69.91 \mathrm{~d}$ & $316.0 \mathrm{c}$ \\
\hline Open-pollinated-3 & $12.53 \mathrm{a}$ & $3.04 \mathrm{a}$ & $1.79 \mathrm{bc}$ & $9.27 \mathrm{~b}$ & $20.05 \mathrm{c}$ & $50.55 \mathrm{~b}$ & $17.48 \mathrm{a}$ & $28.53 \mathrm{~b}$ & $11.78 \mathrm{ab}$ & $9.03 \mathrm{bcd}$ & $5.02 \mathrm{~d}$ & $71.70 \mathrm{~b}$ & $326.6 \mathrm{~b}$ \\
\hline Open-pollinated-4 & $11.81 \mathrm{de}$ & $2.92 \mathrm{~b}$ & $1.74 \mathrm{c}$ & $9.13 \mathrm{~b}$ & $21.55 \mathrm{a}$ & $48.34 \mathrm{c}$ & $15.97 \mathrm{c}$ & $27.57 \mathrm{~d}$ & $11.96 \mathrm{ab}$ & $9.43 \mathrm{a}$ & $5.56 \mathrm{~b}$ & $71.36 \mathrm{bc}$ & $345.3 \mathrm{a}$ \\
\hline Open-pollinated-5 & $11.62 \mathrm{e}$ & $2.85 \mathrm{c}$ & $1.77 \mathrm{c}$ & $9.76 \mathrm{a}$ & $20.94 \mathrm{~b}$ & $53.40 \mathrm{a}$ & $17.21 \mathrm{a}$ & $29.00 \mathrm{a}$ & $11.70 \mathrm{~b}$ & $8.58 \mathrm{e}$ & $5.42 \mathrm{bc}$ & $71.67 \mathrm{~b}$ & $315.9 \mathrm{c}$ \\
\hline Open-pollinated-6 & $11.96 \mathrm{~cd}$ & $3.06 \mathrm{a}$ & $1.88 \mathrm{~b}$ & $9.84 \mathrm{a}$ & $20.12 \mathrm{c}$ & $48.23 \mathrm{c}$ & $15.96 \mathrm{c}$ & $25.18 \mathrm{e}$ & $11.88 \mathrm{ab}$ & $9.20 \mathrm{ab}$ & $5.29 \mathrm{bcd}$ & $70.97 \mathrm{c}$ & $327.9 \mathrm{~b}$ \\
\hline $\mathrm{F}_{1}$ Costanza & $12.28 \mathrm{~b}$ & $3.04 \mathrm{a}$ & $1.86 \mathrm{~b}$ & $9.31 \mathrm{~b}$ & $21.55 \mathrm{a}$ & $50.57 \mathrm{~b}$ & $16.17 \mathrm{c}$ & $28.06 \mathrm{c}$ & $12.04 \mathrm{a}$ & $8.87 \mathrm{~cd}$ & $5.50 \mathrm{bc}$ & $71.66 \mathrm{~b}$ & $347.5 \mathrm{a}$ \\
\hline \multicolumn{14}{|l|}{ Environment $A$} \\
\hline Open-pollinated-1 & $12.27 \mathrm{a}$ & $2.90 \mathrm{a}$ & $1.81 \mathrm{ab}$ & $9.32 \mathrm{de}$ & $19.00 \mathrm{~d}$ & $51.68 \mathrm{~b}$ & $17.25 \mathrm{ab}$ & $31.17 \mathrm{a}$ & $10.85 \mathrm{~b}$ & $8.91 \mathrm{ab}$ & $5.59 \mathrm{ab}$ & $67.14 \mathrm{c}$ & $284.2 \mathrm{c}$ \\
\hline Open-pollinated-2 & $12.50 \mathrm{a}$ & $2.66 \mathrm{c}$ & $1.68 \mathrm{~cd}$ & $9.04 \mathrm{e}$ & $21.10 \mathrm{c}$ & $49.49 \mathrm{~d}$ & $16.56 \mathrm{~cd}$ & $28.21 \mathrm{c}$ & $11.76 \mathrm{a}$ & $9.25 \mathrm{a}$ & $5.31 \mathrm{bc}$ & $66.66 \mathrm{c}$ & $284.2 \mathrm{c}$ \\
\hline Open-pollinated-3 & $12.59 \mathrm{a}$ & $2.81 \mathrm{ab}$ & $1.70 \mathrm{~cd}$ & $9.62 \mathrm{~cd}$ & $18.93 \mathrm{~d}$ & $50.91 \mathrm{bc}$ & $17.72 \mathrm{a}$ & $29.94 \mathrm{~b}$ & $11.28 \mathrm{ab}$ & $9.09 \mathrm{a}$ & $4.96 \mathrm{c}$ & $69.14 \mathrm{ab}$ & $297.5 \mathrm{bc}$ \\
\hline Open-pollinated-4 & $11.27 \mathrm{c}$ & $2.53 \mathrm{~d}$ & $1.65 \mathrm{~d}$ & $9.60 \mathrm{~cd}$ & $24.22 \mathrm{a}$ & $51.45 \mathrm{~b}$ & $16.83 \mathrm{bcd}$ & $30.52 \mathrm{ab}$ & $10.85 \mathrm{~b}$ & $9.22 \mathrm{a}$ & $6.02 \mathrm{a}$ & $69.39 \mathrm{a}$ & $289.2 \mathrm{c}$ \\
\hline Open-pollinated-5 & $12.27 \mathrm{a}$ & $2.75 \mathrm{bc}$ & $1.67 \mathrm{~cd}$ & $9.99 \mathrm{ab}$ & $21.98 \mathrm{c}$ & $53.91 \mathrm{a}$ & $16.94 \mathrm{bc}$ & $30.06 \mathrm{~b}$ & $11.00 \mathrm{~b}$ & $8.57 \mathrm{~b}$ & $5.49 \mathrm{abc}$ & $68.62 \mathrm{ab}$ & $283.3 \mathrm{c}$ \\
\hline Open-pollinated-6 & $11.63 \mathrm{~b}$ & $2.79 \mathrm{~b}$ & $1.89 \mathrm{a}$ & $10.30 \mathrm{a}$ & $21.10 \mathrm{c}$ & $50.13 \mathrm{~cd}$ & $16.28 \mathrm{~d}$ & $28.16 \mathrm{c}$ & $11.70 \mathrm{a}$ & $9.06 \mathrm{a}$ & $5.58 \mathrm{ab}$ & $67.04 \mathrm{c}$ & $312.9 \mathrm{ab}$ \\
\hline $\mathrm{F}_{1}$ Costanza & $12.314 \mathrm{a}$ & $2.82 \mathrm{ab}$ & $1.76 \mathrm{bc}$ & $9.81 \mathrm{bc}$ & $22.17 \mathrm{~b}$ & $49.23 \mathrm{~d}$ & $15.41 \mathrm{e}$ & $28.16 \mathrm{c}$ & $10.81 \mathrm{~b}$ & $9.16 \mathrm{a}$ & $5.75 \mathrm{ab}$ & $67.90 \mathrm{bc}$ & $326.7 \mathrm{a}$ \\
\hline \multicolumn{14}{|l|}{ Environment $B$} \\
\hline Open-pollinated-1 & $12.08 \mathrm{~b}$ & $3.23 \mathrm{a}$ & $1.81 \mathrm{c}$ & $9.02 \mathrm{bcd}$ & $20.97 b$ & $43.92 \mathrm{f}$ & $14.59 \mathrm{c}$ & $23.94 \mathrm{e}$ & $12.64 \mathrm{~b}$ & $8.68 \mathrm{c}$ & $4.78 \mathrm{c}$ & $77.32 \mathrm{a}$ & $355.2 \mathrm{bc}$ \\
\hline Open-pollinated-2 & $11.23 \mathrm{c}$ & $3.13 \mathrm{~b}$ & $2.24 \mathrm{a}$ & $9.19 \mathrm{abc}$ & $22.02 \mathrm{a}$ & $51.04 \mathrm{bc}$ & $17.03 \mathrm{a}$ & $26.04 \mathrm{c}$ & $10.99 \mathrm{~d}$ & $8.87 \mathrm{c}$ & $6.54 \mathrm{a}$ & $73.15 \mathrm{e}$ & $347.9 \mathrm{c}$ \\
\hline Open-pollinated-3 & $12.46 \mathrm{a}$ & $3.27 \mathrm{a}$ & $1.88 \mathrm{bc}$ & $8.93 \mathrm{~cd}$ & $21.16 \mathrm{~b}$ & $50.19 \mathrm{c}$ & $17.23 \mathrm{a}$ & $27.13 \mathrm{~b}$ & $12.29 \mathrm{bc}$ & $8.96 \mathrm{bc}$ & $5.08 \mathrm{bc}$ & $74.26 \mathrm{~cd}$ & $355.6 \mathrm{bc}$ \\
\hline Open-pollinated-4 & $12.35 \mathrm{ab}$ & $3.31 \mathrm{a}$ & $1.84 \mathrm{bc}$ & $8.66 \mathrm{~d}$ & $18.87 \mathrm{~d}$ & $45.22 \mathrm{e}$ & $15.14 \mathrm{~b}$ & $24.61 \mathrm{~d}$ & $13.06 \mathrm{a}$ & $9.63 \mathrm{a}$ & $5.10 \mathrm{bc}$ & $73.32 \mathrm{de}$ & $401.5 \mathrm{a}$ \\
\hline Open-pollinated-5 & $10.29 \mathrm{c}$ & $2.95 \mathrm{c}$ & $1.87 \mathrm{bc}$ & $9.53 \mathrm{a}$ & $19.89 \mathrm{c}$ & $52.89 \mathrm{a}$ & $17.47 \mathrm{a}$ & $27.94 \mathrm{a}$ & $12.39 \mathrm{bc}$ & $8.59 \mathrm{c}$ & $5.34 \mathrm{~b}$ & $74.72 \mathrm{bc}$ & $348.5 \mathrm{c}$ \\
\hline Open-pollinated-6 & $12.29 \mathrm{ab}$ & $3.32 \mathrm{a}$ & $1.86 \mathrm{bc}$ & $9.38 \mathrm{ab}$ & $19.14 \mathrm{~d}$ & $46.33 \mathrm{~d}$ & $15.64 \mathrm{~b}$ & $22.20 \mathrm{f}$ & $12.05 \mathrm{c}$ & $9.33 \mathrm{ab}$ & $5.00 \mathrm{bc}$ & $74.90 \mathrm{bc}$ & $343.0 \mathrm{c}$ \\
\hline $\mathrm{F}_{1}$ Costanza & $12.25 \mathrm{ab}$ & $3.27 \mathrm{a}$ & $1.97 \mathrm{~b}$ & $8.81 \mathrm{~cd}$ & $20.92 \mathrm{~b}$ & $51.91 \mathrm{ab}$ & $16.94 \mathrm{a}$ & $27.96 \mathrm{a}$ & $13.26 \mathrm{a}$ & $8.58 \mathrm{c}$ & $5.25 \mathrm{~b}$ & $75.41 \mathrm{~b}$ & $368.4 \mathrm{~b}$ \\
\hline
\end{tabular}

Note. Distinct letters in each column indicate significant differences according to Duncan's test $(\mathrm{P} \leq 0.05)$. 
Table 3. Statistics (genetic variability- $\sigma^{2} \mathrm{~g}$, phenotypic variability- $\sigma^{2} \mathrm{p}$, genotypic variability coefficient-GCV, phenotypic variability coefficient-PCV, broad sense heritability- $\mathrm{H}$, expected genetic advance-GA, genetic advance as percentage of mean-GA \% mean, Coefficient of Variation-CV, Inter-location total Sum of Squares-SS, GEI is the interaction proportion of total SS) calculated for each of the 13 characteristics (Moisture, Seed oil, Ash content, Seed protein, Ear length, Ear diameter, Number of grain rows per ear, Spindle diameter, Seed length, width and thickness, Hectoliter weight, Thousand kernel weight)

\begin{tabular}{|c|c|c|c|c|c|c|c|c|c|c|c|c|c|}
\hline & Moisture & $\begin{array}{l}\text { Seed } \\
\text { oil }\end{array}$ & $\begin{array}{l}\text { Ash } \\
\text { content }\end{array}$ & $\begin{array}{l}\text { Seed } \\
\text { protein }\end{array}$ & $\begin{array}{l}\text { Ear } \\
\text { length }\end{array}$ & $\begin{array}{l}\text { Ear } \\
\text { diameter }\end{array}$ & $\begin{array}{l}\text { Number of } \\
\text { grain rows } \\
\text { per ear }\end{array}$ & $\begin{array}{l}\text { Spindle } \\
\text { diameter }\end{array}$ & $\begin{array}{l}\text { Seed } \\
\text { length }\end{array}$ & $\begin{array}{l}\text { Seed } \\
\text { width }\end{array}$ & $\begin{array}{l}\text { Seed } \\
\text { thickness }\end{array}$ & $\begin{array}{l}\text { Hectoliter } \\
\text { weight }\end{array}$ & $\begin{array}{l}\text { Thousand- } \\
\text { kernel } \\
\text { weight }\end{array}$ \\
\hline \multicolumn{14}{|l|}{ Environment $A$} \\
\hline$\sigma^{2} g$ & 0.217 & 0.014 & $6.637^{-0.3}$ & 0.159 & 3.373 & 2.418 & 0.512 & 1.516 & 0.139 & 0.041 & 0.069 & 0.968 & 237.51 \\
\hline$\sigma^{2} p$ & 0.231 & 0.015 & $7.892^{-0.3}$ & 0.174 & 3.443 & 2.559 & 0.55 & 1.601 & 0.166 & 0.055 & 0.111 & 1.179 & 285.48 \\
\hline $\mathrm{H}$ & 0.94 & 0.93 & 0.84 & 0.91 & 0.98 & 0.94 & 0.93 & 0.95 & 0.84 & 0.74 & 0.62 & 0.82 & 0.83 \\
\hline GCV & 3.85 & 4.30 & 4.69 & 4.13 & 8.66 & 3.05 & 4.28 & 4.18 & 3.34 & 2.23 & 4.75 & 1.45 & 5.19 \\
\hline PCV & 3.97 & 4.45 & 5.11 & 4.32 & 8.75 & 3.14 & 4.44 & 4.30 & 3.64 & 2.60 & 6.03 & 1.60 & 5.69 \\
\hline GA & 0.931 & 0.235 & 0.154 & 0.782 & 3.75 & 3.1 & 0.14 & 2.48 & 0.705 & 0.358 & 0.425 & 1.834 & 28.89 \\
\hline $\mathrm{GA} \%$ & 7.68 & 8.53 & 8.85 & 8.09 & 17.66 & 6.08 & 8.50 & 8.41 & 6.31 & 3.96 & 7.70 & 2.70 & 9.72 \\
\hline $\mathrm{CV}$ & 7.1 & 8.4 & 13.1 & 8.6 & 11.0 & 5.3 & 8.1 & 7.2 & 9.5 & 8.4 & 22.9 & 4.34 & 15 \\
\hline \multicolumn{14}{|l|}{ Environment B } \\
\hline$\sigma^{2} g$ & 0.339 & 0.0165 & 0.0196 & 0.0776 & 1.265 & 12.54 & 1.28 & 4.75 & 0.542 & 0.141 & 0.312 & 1.850 & 376.33 \\
\hline$\sigma^{2} p$ & 0.349 & 0.0173 & 0.0217 & 0.0963 & 1.327 & 12.68 & 1.31 & 4.80 & 0.562 & 0.159 & 0.331 & 1.980 & 400.89 \\
\hline $\mathrm{H}$ & 0.97 & 0.95 & 0.90 & 0.80 & 0.95 & 0.99 & 0.97 & 0.99 & 0.96 & 0.89 & 0.94 & 0.93 & 0.94 \\
\hline $\mathrm{GCV}$ & 4.87 & 4.0 & 7.28 & 3.070 & 5.51 & 7.26 & 6.94 & 8.49 & 5.95 & 4.19 & 10.54 & 1.82 & 5.39 \\
\hline PCV & 4.94 & 4.09 & 7.66 & 3.42 & 5.64 & 7.30 & 7.04 & 8.53 & 6.06 & 4.45 & 10.86 & 1.88 & 5.56 \\
\hline GA & 1.18 & 0.257 & 0.273 & 0.511 & 2.254 & 7.262 & 2.29 & 4.47 & 1.48 & 0.73 & 1.114 & 2.696 & 38.77 \\
\hline GA $\%$ & 9.87 & 8.02 & 14.19 & 5.63 & 11.04 & 14.88 & 14.06 & 17.43 & 11.97 & 8.17 & 21.01 & 3.61 & 10.77 \\
\hline $\mathrm{CV}$ & 6.8 & 6.5 & 15.6 & 9.4 & 8.9 & 8.1 & 9.5 & 9.4 & 8.7 & 9.7 & 18.2 & 3.3 & 9.6 \\
\hline \multicolumn{14}{|l|}{ Inter-location SS } \\
\hline Location (E) & 2 & 79 & 41 & 43 & 7 & 16 & 5 & 56 & 54 & 2 & 7 & 89 & 77 \\
\hline Genotype (G) & 33 & 10 & 22 & 38 & 22 & 42 & 43 & 21 & 6 & 70 & 36 & 4 & 12 \\
\hline Interaction (GEI) & 65 & 11 & 37 & 19 & 71 & 42 & 52 & 23 & 40 & 28 & 57 & 7 & 11 \\
\hline
\end{tabular}

In environment $\mathrm{B}$, almost all characteristics showed high heritability that reached 0.99 for ear and spindle diameter, 0.97 for moisture and number of grain rows, 0.96 for seed length, 0.95 for seed oil and ear length, 0.94 for seed thickness and thousand-kernel weight and, 0.93 for hectoliter weight. GCV and PCV values were greater for ear length in environment A (over 8), while in environment B, GCV and PCV values were greater for seed thickness, ear and spindle diameter and ash content. In environment A, genetic advance has been calculated and found high only for two characteristics, while in environment B for seven. Seed thickness showed the greatest experimental CV in both environments, near or greater than $20 \%$. Concerning sum of squares (SS), environment showed the greatest proportion in the case of hectoliter weight, thousand-kernel weight, seed oil, spindle diameter and seed length (also found for ash content and seed protein). Genotype contributed the greatest proportion only for seed width $(70 \%)$, while interaction was significant for ear length, moisture, seed thickness and number of grain rows.

Descriptive statistics (Table 4) showed that inter-location range ranged from 10.8 to $12.9 \%$ for moisture, from 2.5 to $3.4 \%$ for seed oil, from 1.3 to $2.1 \%$ for ash content, from 7.7 to $10.8 \%$ for seed protein, from 80.8 to 84.4 for $\mathrm{L}^{*}$, from -0.89 to 1.17 for $\mathrm{a}^{*}$, from 16.98 to $30 \mathrm{for}^{*}$, from 17.7 to $24.3 \mathrm{~cm}$ for ear length, from 41.2 to 54.8 $\mathrm{mm}$ for ear diameter, from 14 to 18 for number of grain rows per ear, from 20.9 to $31.2 \mathrm{~mm}$ for spindle diameter, from 9.17 to $13.79 \mathrm{~mm}$ for seed length, from 7.89 to $10.66 \mathrm{~mm}$ for seed width, from 4.48 to $8.92 \mathrm{~mm}$ for seed thickness, from 66.66 to $78.19 \mathrm{~kg} \mathrm{~L}^{-1}$ for hectoliter weight, from 284.2 to $411.5 \mathrm{~g}$ for TKW. Seed thickness showed the greatest $\mathrm{CV}$ value $24.6 \%$. 
Table 4. Descriptive statistics (Inter-location data): minimum, maximum, mean, standard deviation and coefficient of variation (CV) for the 17 quality and quantity characteristics (Moisture, Seed oil, Ash content, Seed protein, Ear length, Ear diameter, Number of grain rows per ear, Spindle diameter, Seed length, width and thickness, Hectoliter weight, Thousand kernel weight, $\mathrm{pH}$ and color characteristics $\mathrm{L}^{*}, \mathrm{a}^{*}, \mathrm{~b}^{*}$, corresponding to luminosity, redness and yellowness, respectively)

\begin{tabular}{llllll}
\hline Variable & Minimum & Maximum & Mean & Std. deviation & CV (\%) \\
\hline Moisture & 10.792 & 12.903 & 12.126 & 0.661 & 5.1 \\
Seed oil & 2.526 & 3.415 & 2.976 & 0.275 & 9.2 \\
Ash content & 1.292 & 2.108 & 1.814 & 0.205 & 11.3 \\
Seed protein & 7.697 & 10.766 & 9.147 & 0.833 & 9.1 \\
$\mathrm{pH}$. & 6.34 & 6.596 & 6.499 & 0.073 & 1.1 \\
$\mathrm{~L}^{*}$ & 80.822 & 84.371 & 82.768 & & \\
$\mathrm{a}^{*}$ & -0.886 & 1.17 & 0.144 & & \\
$\mathrm{~b}^{*}$ & 16.976 & 30.002 & 24.467 & & \\
Ear length & 17.7 & 24.3 & 20.466 & 2.084 & 10.2 \\
Ear diameter & 41.21 & 54.8 & 49.435 & 3.933 & 7.9 \\
Number of grain rows per ear & 14 & 18 & 16.25 & 1.66 & 10.2 \\
Spindle diameter & 20.92 & 31.166 & 27.528 & 3.156 & 11.5 \\
Seed length & 9.17 & 13.79 & 11.491 & 1.373 & 11.9 \\
Seed width & 7.89 & 10.66 & 9.002 & 0.703 & 7.8 \\
Seed thickness & 4.48 & 8.92 & 5.521 & 1.357 & 24.6 \\
Hectoliter weight & 66.657 & 78.19 & 71.787 & 3.9 & 5.4 \\
Thousand-kernel weight & 284.15 & 411.5 & 342.86 & 38.781 & 11.3 \\
\hline
\end{tabular}

Correlations (Table 5) showed that seed oil content $(\%)$ was negatively correlated $(r=-0.569)$ to seed protein content $(\%)$. The $\mathrm{pH}$ values were positively correlated to moisture and seed oil content, while the color parameters $\mathrm{L}^{*}$ and $\mathrm{a}^{*}$, corresponding to luminosity and redness, respectively, were negatively correlated $(\mathrm{r}=$ -0.851). Ear diameter was positively correlated to spindle diameter and number of grain rows. Seed oil was negatively correlated to spindle diameter. Hectoliter weight was positively correlated to seed oil and TKW, but negatively to spindle diameter. 
Table 5. Correlation coefficients between the 17 quantity and quality characteristics (Moisture, Seed oil, Ash content, Seed protein, Ear length, Ear diameter, Number of grain rows per ear, Spindle diameter, Seed length, width and thickness, Hectoliter weight, Thousand kernel weight, $\mathrm{pH}$ and color characteristics $\mathrm{L}^{*}, \mathrm{a}^{*}, \mathrm{~b}^{*}$, corresponding to luminosity, redness and yellowness, respectively)

\begin{tabular}{|c|c|c|c|c|c|c|c|c|c|c|c|c|c|c|c|c|c|}
\hline Variables & $\begin{array}{l}\stackrel{0}{\Xi} \\
.00 \\
\stackrel{n}{\Sigma}\end{array}$ & 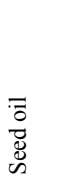 & 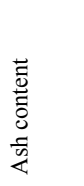 & 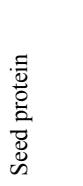 & 咅 & $\stackrel{*}{\bullet}$ & $*_{\sigma}$ & مـ & 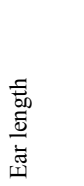 & 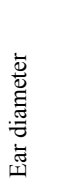 & 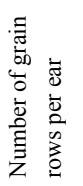 & 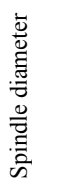 & 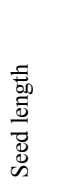 & 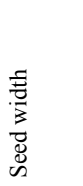 & 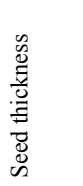 & 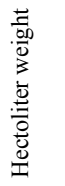 & 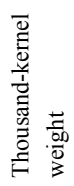 \\
\hline Moisture & 1 & & & & & & & & & & & & & & & & \\
\hline Seed oil & 0.455 & 1 & & & & & & & & & & & & & & & \\
\hline Ash content & -0.152 & 0.174 & 1 & & & & & & & & & & & & & & \\
\hline Seed protein & -0.116 & -0.569 & 0.341 & 1 & & & & & & & & & & & & & \\
\hline $\mathrm{pH}$ & 0.793 & 0.710 & 0.042 & -0.411 & 1 & & & & & & & & & & & & \\
\hline $\mathrm{L}^{*}$ & -0.095 & -0.112 & -0.644 & -0.596 & 0.059 & 1 & & & & & & & & & & & \\
\hline$a^{*}$ & -0.2 & 0.011 & 0.793 & 0.556 & -0.24 & -0.851 & 1 & & & & & & & & & & \\
\hline$b^{*}$ & 0.565 & 0.596 & -0.279 & -0.253 & 0.662 & 0.191 & -0.398 & 1 & & & & & & & & & \\
\hline Ear length & -0.538 & -0.473 & 0.191 & 0.411 & -0.451 & -0.165 & 0.235 & -0.213 & 1 & & & & & & & & \\
\hline Ear diameter & -0.385 & -0.455 & -0.473 & 0.011 & -0.262 & 0.565 & -0.468 & 0.068 & 0.393 & 1 & & & & & & & \\
\hline $\begin{array}{l}\text { Number of grain } \\
\text { rows per ear }\end{array}$ & -0.043 & -0.169 & -0.553 & 0.023 & 0.041 & 0.309 & -0.364 & 0.321 & 0.124 & 0.754 & 1 & & & & & & \\
\hline Spindle diameter & -0.464 & -0.705 & -0.226 & 0.398 & -0.521 & 0.191 & -0.029 & -0.297 & 0.437 & 0.829 & 0.598 & 1 & & & & & \\
\hline Seed length & 0.178 & 0.648 & 0.244 & -0.336 & 0.473 & -0.174 & 0.266 & 0.288 & -0.358 & -0.389 & 0.05 & -0.455 & 1 & & & & \\
\hline Seed width & 0.275 & -0.266 & -0.323 & -0.055 & 0.077 & 0.182 & -0.420 & -0.209 & -0.42 & -0.059 & -0.045 & -0.007 & -0.240 & 1 & & & \\
\hline Seed thickness & -0.224 & -0.185 & -0.013 & -0.084 & -0.185 & 0.165 & -0.064 & -0.216 & 0.178 & 0.145 & -0.226 & 0.255 & -0.207 & 0.315 & 1 & & \\
\hline Hectoliter weight & 0.2 & 0.732 & 0.174 & -0.495 & 0.437 & -0.002 & -0.143 & 0.464 & -0.415 & -0.393 & -0.294 & -0.701 & 0.495 & -0.033 & -0.183 & 1 & \\
\hline $\begin{array}{l}\text { Thousand-kernel } \\
\text { weight }\end{array}$ & 0.015 & 0.53 & 0.402 & -0.284 & 0.204 & -0.389 & 0.226 & -0.081 & -0.174 & -0.587 & -0.465 & -0.67 & 0.459 & 0.095 & -0.037 & 0.71 & 1 \\
\hline
\end{tabular}

Note. Values in bold are different from 0 with a significance level alpha $=0.05$.

Cluster analysis (Figure 4) revealed two main clusters, which were formed in relation to the environments. Even Costanza characteristics were different in the two environments. Only characteristics of open-pollinated line 4 were similar in the two different locations.

PCA analysis (Tables 6 and 7 and Figures 5 and 6) showed that the first four variables may interpret almost $80 \%$ of the total variability of characteristics studied. Open-pollinated line four appears on the upper half of the PCA biplot, while open-pollinated line five appears on the lower half (Figure 5). PCA biplot showed that open-pollinated line two from the two different environments is located nearby on the lower right quarter of the biplot and open-pollinated line 3 is located near but in different quarters of lower part of biplot. PCA analysis also depicted that some characteristic showed the greatest contribution in defining the major components variables: Seed thickness, seed length, moisture content, seed width and $\mathrm{a}^{*}$. Ear and spindle diameter were located nearby and $\mathrm{pH}$ with moisture content also and very close to seed oil content and hectoliter weight which were almost on the same spot in the biplot of the two main variables.

Table 6. Eigenvalues of each principal component and the proportion (\%) of the total variability explained by each variable

\begin{tabular}{llllllllllllll}
\hline & F1 & F2 & F3 & F4 & F5 & F6 & F7 & F8 & F9 & F10 & F11 & F12 & F13 \\
\hline Eigenvalue & 5.617 & 4.029 & 2.025 & 1.625 & 0.938 & 0.852 & 0.689 & 0.414 & 0.404 & 0.268 & 0.076 & 0.052 & 0.013 \\
Variability (\%) & 33.044 & 23.698 & 11.910 & 9.559 & 5.515 & 5.011 & 4.050 & 2.434 & 2.376 & 1.576 & 0.444 & 0.304 & 0.078 \\
Cumulative \% & 33.044 & 56.742 & 68.652 & 78.211 & 83.726 & 88.737 & 92.788 & 95.221 & 97.598 & 99.174 & 99.618 & 99.922 & 100.000
\end{tabular}


Table 7. First six components from the PCA analysis of 17 quantity and quality characteristics (Moisture, Seed oil, Ash content, Seed protein, Ear length, Ear diameter, Number of grain rows per ear, Spindle diameter, Seed length, width and thickness, Hectoliter weight, Thousand kernel weight, $\mathrm{pH}$ and color characteristics $\mathrm{L}^{*}, \mathrm{a}^{*}, \mathrm{~b}^{*}$, corresponding to luminosity, redness and yellowness, respectively) with the contribution (\%) of each characteristic to total variability

\begin{tabular}{lllllll}
\hline & F1 & F2 & F3 & F4 & F5 & F6 \\
\hline Moisture & 5.687 & 2.813 & 0.278 & 30.030 & 3.429 & 0.077 \\
Seed oil & 14.089 & 0.509 & 2.444 & 2.238 & 2.087 & 0.434 \\
Ash content & 1.340 & 16.561 & 0.601 & 0.361 & 4.399 & 0.657 \\
Seed protein & 3.737 & 8.278 & 2.987 & 15.786 & 0.01 & 0.118 \\
pH. & 9.088 & 3.894 & 2.268 & 4.253 & 8.538 & 2.345 \\
L* & 0.890 & 16.374 & 2.395 & 6.326 & 0.010 & 1.535 \\
a* & 0.088 & 21.103 & 3.249 & 0.310 & 0.000 & 4.849 \\
b* & 3.580 & 7.869 & 12.382 & 0.474 & 9.924 & 2.323 \\
Ear length & 5.984 & 3.143 & 3.980 & 5.278 & 12.193 & 5.033 \\
Ear diameter & 9.436 & 6.262 & 3.215 & 3.822 & 0.468 & 1.393 \\
Number of grain rows per ear & 2.943 & 7.702 & 14.986 & 0.016 & 5.331 & 13.282 \\
Spindle diameter & 14.501 & 0.196 & 1.663 & 0.000 & 0.115 & 10.186 \\
Seed length & 7.878 & 0.052 & 4.999 & 3.402 & 3.323 & 30.256 \\
Seed width & 0.018 & 2.011 & 26.592 & 11.283 & 1.471 & 8.939 \\
Seed thickness & 1.188 & 0.006 & 14.007 & 2.268 & 46.059 & 16.011 \\
Hectoliter weight & 11.354 & 0.297 & 0.175 & 7.852 & 0.403 & 1.475 \\
Thousand-Kernel weight & 8.200 & 2.931 & 3.777 & 6.303 & 2.240 & 1.087 \\
Total & 100 & 100 & 100 & 100 & 100 & 100 \\
\hline
\end{tabular}

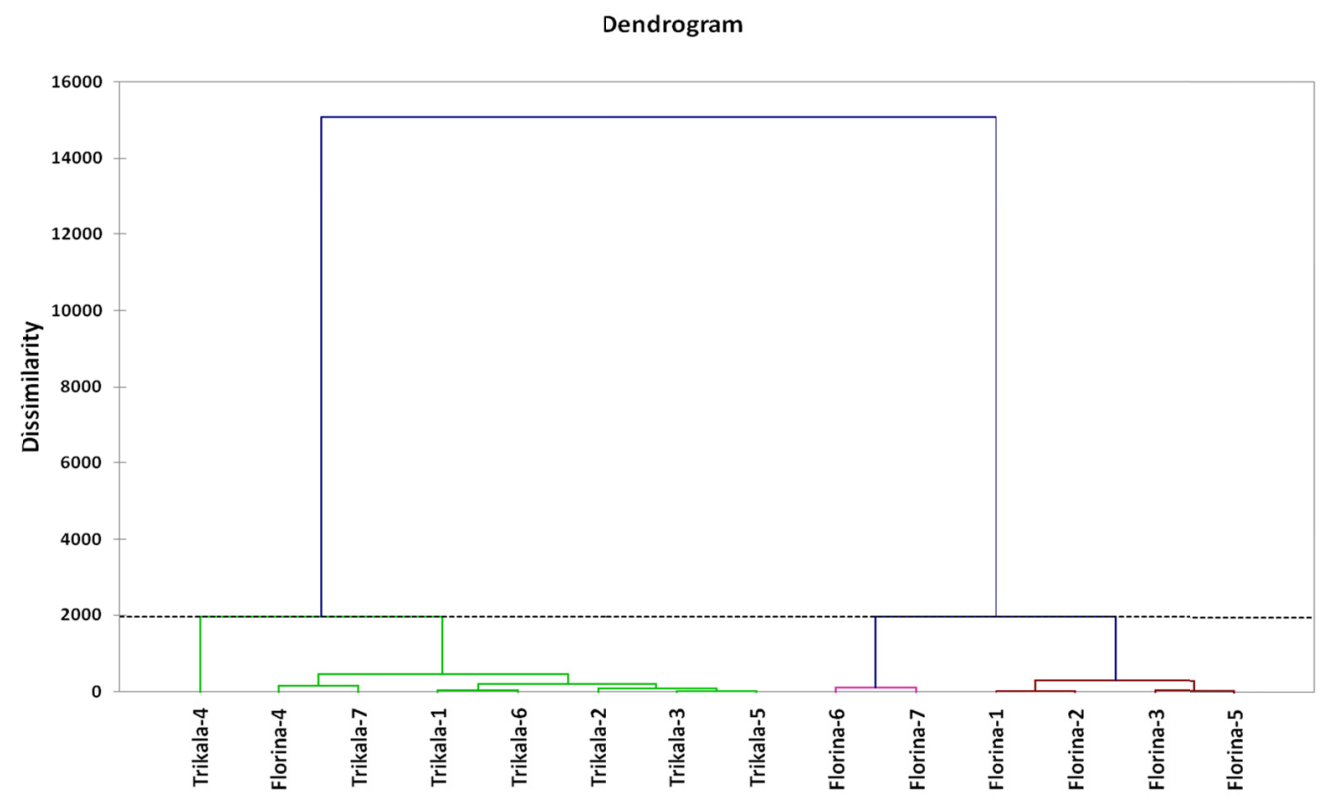

Figure 4. Dendrogram using agglomerative hierarchical clustering (AHC) for maize genetic materials based on 17 quantity and quality characteristics (Moisture, Seed oil, Ash content, Seed protein, Ear length, Ear diameter, Number of grain rows per ear, Spindle diameter, Seed length, width and thickness, Hectoliter weight, Thousand kernel weight, $\mathrm{pH}$ and color characteristics $\mathrm{L}^{*}, \mathrm{a}^{*}, \mathrm{~b}^{*}$, corresponding to luminosity, redness and yellowness, respectively) 


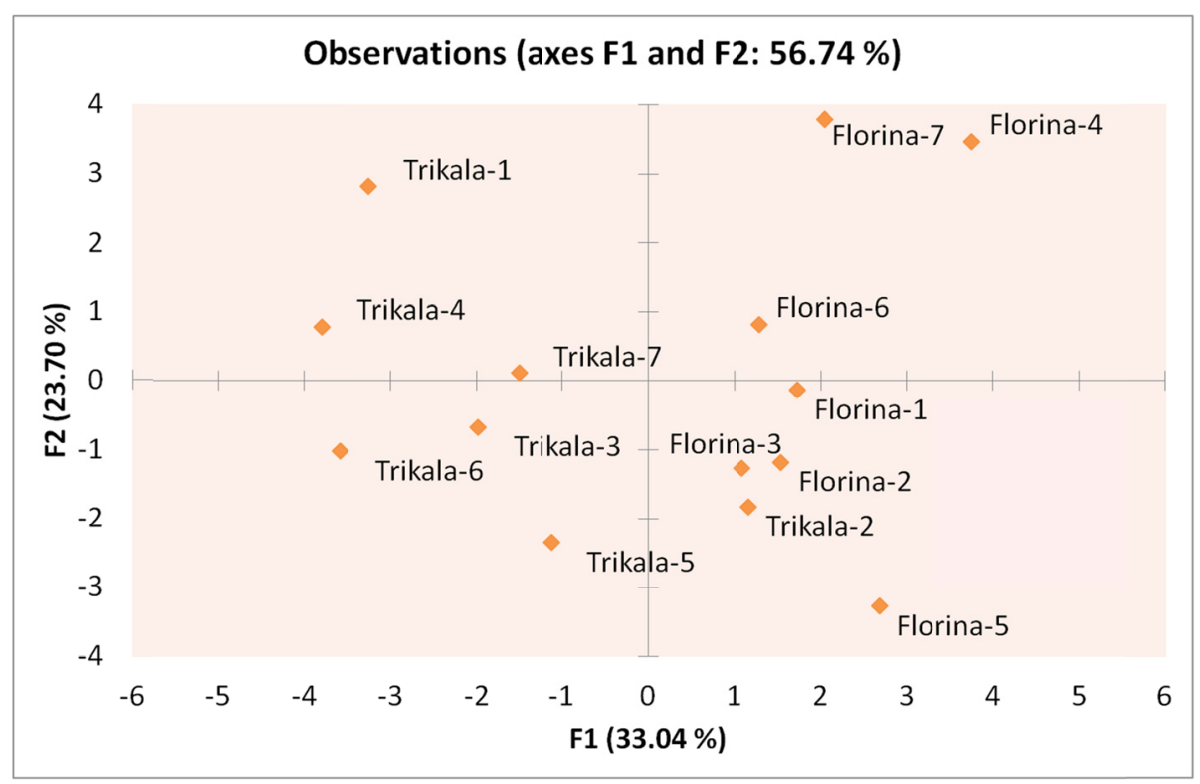

Figure 5. Principal Component Analysis of 17 quantity and quality characteristics (Moisture, Seed oil, Ash content, Seed protein, Ear length, Ear diameter, Number of grain rows per ear, Spindle diameter, Seed length, width and thickness, Hectoliter weight, Thousand kernel weight, $\mathrm{pH}$ and color characteristics $\mathrm{L}^{*}, \mathrm{a}^{*}, \mathrm{~b}^{*}$, corresponding to luminosity, redness and yellowness, respectively) and two-dimensional distribution of the genetic materials

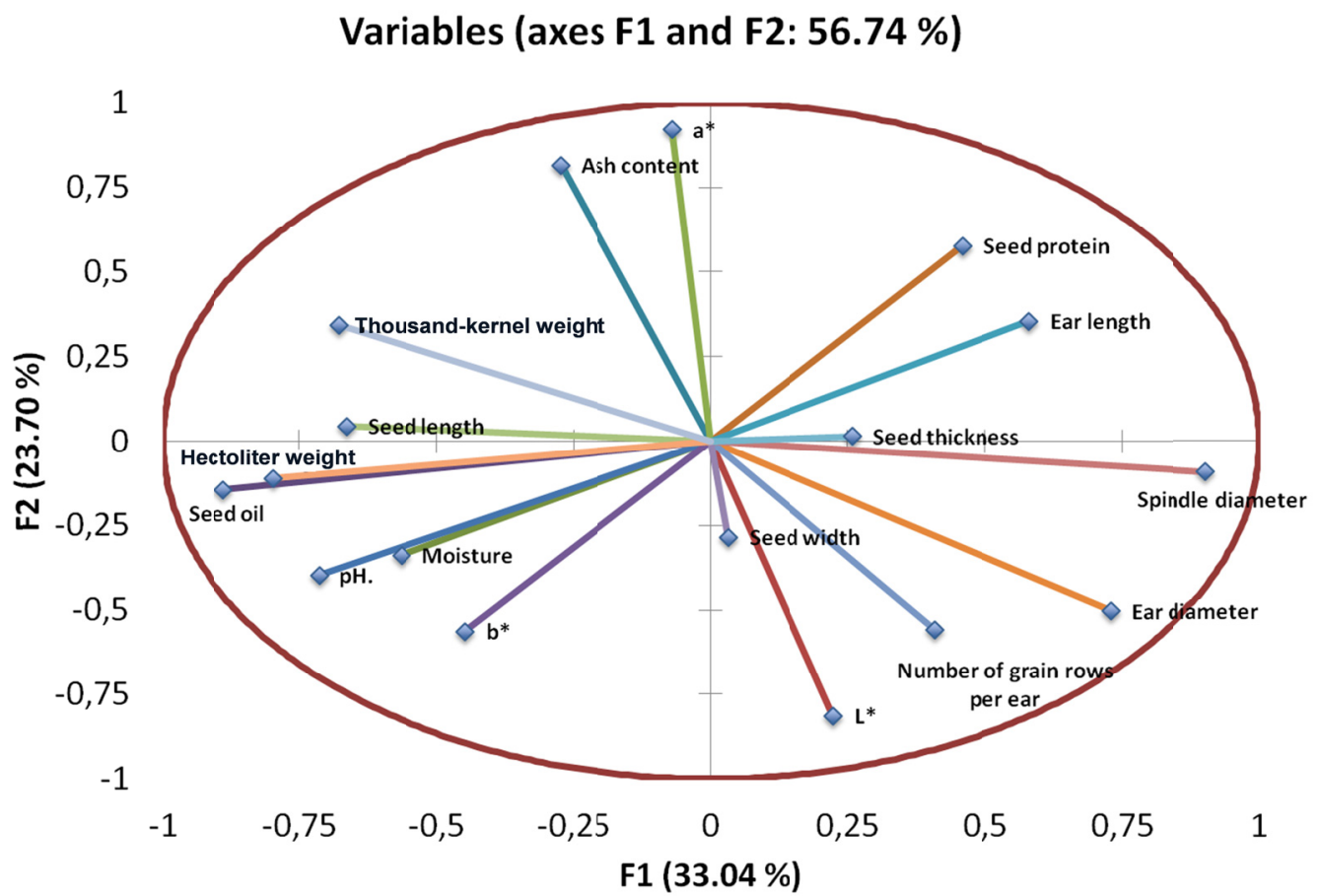

Figure 6. Principal Component Analysis of genetic diversity of maize genetic materials and distribution of the 17 quantity and quality characteristics (Moisture, Seed oil, Ash content, Seed protein, Ear length, Ear diameter, Number of grain rows per ear, Spindle diameter, Seed length, width and thickness, Hectoliter weight, Thousand kernel weight, $\mathrm{pH}$ and color characteristics $\mathrm{L}^{*}, \mathrm{a}^{*}, \mathrm{~b}^{*}$, corresponding to luminosity, redness and yellowness, respectively) 


\section{Discussion}

Limited diversity in maize may lead to stagnation and genetic vulnerability to biotic and abiotic stresses, thus open-pollinated varieties and local populations may be of great importance for breeding purposes (Taller \& Bernardo, 2004).

Heritability of traits is of great importance for plant breeders, because they have to decide the selection procedure to be followed for coping with a certain breeding situation (Li \& Yang, 1985). However, high heritability accompanied by high genetic advance would be more reliable for formulating successful selection procedure (Nataraj, Shahi, \& Vandana, 2014). Combination of these two important parameters is of great importance for predicting gains under the selected procedures (Sharma, Punia, \& Kamboj, 2015). Johnson, Robinson, and Comstock (1955), exploring gene action, were the first who depicted the effectiveness of the use of genetic advance in combination to heritability estimates, because of the fixation of useful additive genes under the selected breeding procedure.

Our data suggest that moisture content, seed oil content, ear length, ear diameter, number of grain rows, spindle diameter and seed thickness exhibit inter-location stability and high heritability. Seed protein, seed length, TKW, hectoliter weight and seed thickness heritability is highly depended on the environment, especially environment B (Trikala), which seems to favor heritability. Seed thickness heritability was influenced strongly by environment, because in environment B presented high heritability, while in environment A showed the lowest one. In environment A only TKW and ear length showed high genetic advance, but in environment B, ear length and TKW, ear diameter, number of rows, spindle diameter, and seed length and thickness showed high genetic advance, revealing also favorable conditions of environment B. According to the previously mentioned studies, high heritability and high genetic advance indicate strong additive gene action that may ensure gains through selection procedure (Sharma, Punia, \& Kamboj, 2015). High PCV accompanied by high GCV reveals significant differentiation and genetic expression (Kinfe \& Tsehaye, 2015), found in five cases: for ear length in environment $\mathrm{A}$, for ash content, ear diameter, spindle diameter and seed thickness. Seed thickness showed a rather unstable behavior because of the high CV value. Generally, environment and genotype by environment interaction contributed the greatest proportion of variability (inter-location SS calculations) for almost all characteristics studied except for seed width where genetic variability is the greatest proportion of total variability. Honeycomb design although records the relation between local phenotype behavior and genotype contribution resulting in high heritability, is unable to record locality effects when genetic materials are grown in different environments. For this reason, Sum of Squares analysis is an additional tool that may reveal inter-location environmental effects and interactions and may unmask the real phenotypic variability.

Summarizing, high heritability of certain characteristics in our study, accompanied by high genetic advance and high GCV, indicate heritable additive gene action and high contribution of genotypes. This was found for some cases like ear diameter, ear length, spindle diameter and number of grain rows. Nataraj, Shahi, and Vandana (2014) reported similar results. Seed thickness showed contrasting results and unstable behavior. TKW showed satisfactory results and in some cases a few more characteristics. SS estimations in our study seem to be in contrast to the statement that high heritability, genetic advance and GCV reveal high genetic contribution. Local environment affects strongly variation and genotype by environment interaction is present in many cases. Seed width, although it did not exhibit high heritability, genetic advance and GCV, was the only characteristic to be depended on genetic variability according to SS estimations. We believe that for many characteristics (included in our study), genetic contribution is truly high, reflected in the parameters studied and resulting in high heritability and, subsequently, in success of a certain breeding program, but environment to be practiced such a program must be properly chosen (Greveniotis et al., 2009). Environment B (Trikala) seems to be most proper for breeding purposes boosting genetic expression and heritability of many characteristics. In many cases means were better and better distinguished to each other under comparisons, accompanied by high and statistically significant differences.

Descriptive statistics revealed a great range of mean fluctuations, indicating that there is satisfactory variability in many characteristics to be exploited by breeders. Some characteristics showed low CV values, indicating stability of performance and low environmental effects. Also, correlations found in our study may be a useful tool for indirect selection of certain characteristics difficult to be selected due to non-additive effects (Hallauer, Carena, \& Miranda Filho, 2010). In such cases, correlated selection differential may be used for prediction of indirect selection accuracy (Bos \& Caligari, 2008). High heritability of ear characteristics found in this study could be used as a means for improving yield, proposed also by other researchers (Ross, 2002; Ghimire \& Timsina, 2015). Many reports on certain traits' correlations, not always in agreement to each other and our data, were previously performed in maize like the work of Chukwu, Ekwu, Onyishi, Okporie, and Obi (2013), and 
Iqbal, Shinwari, and Rabbani (2015). High negative correlations of seed oil and seed protein found in our study, may lead to different purpose maize varieties through divergent selection. This goal has been achieved in a breeding program in USA for many years (Dudley \& Lambert, 2004). High negative correlations of L* (corresponding to luminosity) and $\mathrm{a}^{*}$ (corresponding to redness) may result in easier color estimations. Estimations of $\mathrm{pH}$ are highly correlated to moisture and seed oil content (in a triple correlation) and may be used for selecting for low kernel moisture and proper seed oil content. Similar results but not for $\mathrm{pH}$ were reported by Scrob et al. (2014). Spindle diameter found to be positively correlated to ear diameter and number of kernel rows [partly referred by Kwaga (2014) and in agreement to Iqbal, Shinwari, and Rabbani (2015)]. Although spindle diameter is not considered a desirable characteristic because, as it is positively correlated to total ear diameter, kernel depth remains low, sometimes may contribute in ear stability (during pre-drying in the field and in machine harvesting) and total ear diameter and subsequently to harvest yield (Larson \& Hanway, 1977; Georgiev \& Mouhtanov, 1980; FAO, 1991). Hectoliter weight is positively correlated to seed oil content and TKW [in agreement to Scrob et al. (2014)] but negatively to spindle diameter. Seed length and spindle diameter are also correlated (positively and negatively respectively) to seed oil content and may be used also for indirect estimations. Color characteristics can be used for seed protein estimations and ear diameter estimations. High a* values, corresponding to redness, may be a result of denser kernels, with high protein content (Jaradat, 2013).

Cluster analysis was used many times for classification purposes of maize genetic materials, sometimes in combination to principal components analysis (Hafiz, Jehanzeb, Ejaz-ul-Hasan, Tahira, \& Tariq, 2015). Cluster analysis of our data set resulted in two main groups, exhibiting that environment of selection may influence classification of open-pollinated maize lines. These lines behaved like different materials even though they were derived from the same C4 mother lines. Even F1 commercial maize hybrid Costanza was classified in different groups. This is an indication of the environmental impact on phenotypic expression. Only open-pollinated line four exhibited related expression of characteristics studied, revealing gene fixation and stability of performance (Fasoulas, 1993).

PCA analysis showed different results in comparison to Cluster analysis. PCA biplot showed that open-pollinated line 2 from the two different environments is located nearby on the lower right quarter of the biplot and open-pollinated line three is located near but in different quarters of lower part of biplot. These results may be due to axis system used based on the first two variables that explain only $57 \%$ of total variability. Open-pollinated line 4 from the two locations, in PCA showed a slightly different behavior. Seed thickness, seed length, moisture content, seed width and $\mathrm{a}^{*}$ showed the greatest contribution in defining the major components variables. Ear and spindle diameter were located nearby and $\mathrm{pH}$ with moisture content also and very close to seed oil content and hectoliter weight which were almost on the same spot in the biplot of the two main variables. Environmental effects were also present in phenotypic expression of the characteristics studied and classification of lines was not the expected one.

\section{Conclusions}

Moisture content, seed oil content, ear length, ear diameter, number of grain rows, spindle diameter and seed thickness exhibit inter-location high heritability but heritability estimations were highly depended on the environment, since GEI interaction was high indicating environmental interaction with genotype, especially environment $B$ which seems to favor heritability. Locality affects strongly variation and genotype by environment interaction is significant in many cases. Seed width was the only characteristic to be depended on genetic variability. Mean fluctuations revealed satisfactory variability in many characteristics to be exploited by breeders. Some characteristics showed low CV values (Moisture, Seed oil, Ear diameter, Spindle diameter, Number of grain row per ear, and Hectoliter weight), indicating stability of performance and low environmental effects. Significant correlations found in our study may be a useful tool for indirect selection of certain characteristics, otherwise difficult to be selected due to non-additive effects. Cluster analysis and PCA showed contrasting results in classification of open-pollinated lines and this was attributed to strong environmental effects that distorted phenotypic expression of the characteristics studied. The investigation of genetic variation in the characteristics determined at the present study and the assessment of possible correlations among variables may be used for breeding purposes. Some correlations found are reasonable like Ear diameter and Spindle diameter or Number of grain rows per ear, also between $\mathrm{L}^{*}$ and $\mathrm{a}^{*}$ color parameters. A few measurements are depended on $\mathrm{pH}$, like Moisture content and Seed oil content. Finally, we can use Spinle diameter, Seed length and Hectoliter weight for indirect improvement of Seed oil content. 


\section{Acknowledgements}

This research work and authors was based on the remarks and support of late professors A.C. Fasoulas, St. Zotis. This research has been partly co-financed by the European Union (European Social Fund-ESF) and Greek national funds through the Operational Program "Education and Lifelong Learning" of the National Strategic Reference Framework (NSRF)-Research Funding Program: Heracleitus II. Investing in knowledge society through the European Social Fund.

The authors thank UNITY IMPORTS EXPORTS LTD for their support during the calibration of SpectraStar, the Near-Infrared (NIR) reflectance spectroscopy instrument, which was performed using UCAL Calibration software

\section{References}

Alves, B. M., Cargnelutti Filho, A., Toebe, M., Burin, C., \& Silva, L. P. (2014). Variability of grain productivity and energy profile of maize (Zea mays L.) genotypes. Journal of Cereal Science, 60, 164-171. https://doi.org/10.1016/j.jcs.2014.02.007

Bos, I., \& Caligari, P. (2008). Selection methods in plant breeding (2nd ed.). Dordrecht, Netherlands, Springer. https://doi.org/10.1007/978-1-4020-6370-1

Chukwu, S. C., Ekwu, L. G., Onyishi, G .C., Okporie, E. O., \& Obi, I. U. (2013). Correlation between agronomic and chemical characteristics of maize (Zea Mays L.) genotypes after two years of mass selection. International Journal of Science and Research, 4(8), 1708-1712.

de Oliveira, E. J., Dias, N. L. P., \& Dantas, J. L. L. (2012). Selection of morpho-agronomic descriptors for characterization of papaya cultivars. Euphytica, 185(2), 253-265. https://doi.org/10.1007/s10681-0110565-0

Dudley, J. W., \& Lambert, R. J. (2004). 100 generations of selection for oil and protein in corn. In J. Janick (Ed.), Plant breeding reviews (Vol. 24, pp. 79-110). John Wiley \& Sons.

FAO. (1991). Agricultural engineering in development-The harvest. Retrieved from http://www.fao.org/docrep/ t0522e/T0522E05.htm

Fasoula, V. A. (2006). A novel equation paves the way for an everlasting revolution with cultivars characterized by high and stable crop yield and quality. Proceedings of the 11th National Hellenic Conference in Genetics and Plant Breeding (pp. 7-14). Greece.

Fasoula, V. A. (2013). Prognostic Breeding: A new paradigm for crop improvement. In J. Janick (Ed.), Plant breeding reviews (Vol. 37, pp. 297-347). John Wiley \& Sons. https://doi.org/10.1002/9781118497869.ch6

Fasoulas, A. C. (1988). The Honeycomb Methodology of Plant Breeding. Department of Genetics and Plant Breeding, Aristotle University of Thessaloniki, Greece.

Fasoulas, A. C. (1993). Principles of crop breeding. Department of Genetics and Plant Breeding, Aristotle University of Thessaloniki, Greece.

Fasoulas, A. C., \& Fasoula, V. A. (1995). Honeycomb selection designs. In J. Janick (Ed.), Plant breeding reviews (Vol. 13, pp. 87-139). John Wiley \& Sons. https://doi.org/10.1002/9780470650059.ch3

Ganopoulos, I., Moysiadis, T., Xanthopoulou, A., Ganopoulou, M., Avramidou, E., Aravanopoulos, F. A., ... Kazantzis, K. (2015). Diversity of morpho-physiological traits in worldwide sweet cherry cultivars of GeneBank collection using multivariate analysis. Scientia Horticulturae, 197, 381-391. https://doi.org/ 10.1016/j.scienta.2015.09.061

Georgiev, T., \& Mouhtanov, I. (1980). Relationships among several characteristics in maize related to the kernel moisture content at harvest. Maize Newsletter Index, 54, 102-105.

Ghimire, B., \& Timsina, D. (2015). Analysis of Yield and Yield Attributing Traits of Maize Genotypes in Chitwan, Nepal. World Journal of Agricultural Research, 3(5), 153-162. https://doi.org/10.12691/ wjar-3-5-2

Greveniotis, V., Xanthopoulou, O., Pessios, E., Deligeorgidis, P., Stefanis, D., \& Ipsilandis, C. G. (2009). Honeycomb evaluation of barley germplasm under pre-evaluated environments. Cereal Research Communications, 37(4), 579-586. https://doi.org/10.1556/CRC.37.2009.4.11

Gustin, J. L., Jackson, S., Williams, C., Patel, A., Armstrong, P., Peter, G. F., \& Settles, A. M. (2013). Analysis of Maize (Zea mays) Kernel Density and Volume Using Microcomputed Tomography and Single-Kernel 
Near-Infrared Spectroscopy. Journal of Agricultural and Food, 61(46), 10872-10880. https://dx.doi.org/ $10.1021 / \mathrm{j} 4403790 \mathrm{v}$

Hafiz, S. B. M., Jehanzeb, F., Ejaz-ul-Hasan, Tahira, B., \& Tariq, M. (2015). Cluster and principle component analyses of maize accessions under normal and water stress conditions. Journal of Agricultural Sciences, 60(1), 33-48. https://doi.org/10.2298/JAS1501033M

Hallauer, A. R., Carena, M. J., \& Miranda Filho, J. B. (2010). Quantitative genetics in maize breeding. In J. Prohens, F. Nuez, \& M. J. Carena (Eds.), Handbook of plant breeding. New York, Springer.

Iqbal, J., Shinwari, Z. K., \& Rabbani, M. A. (2015). Maize (Zea mays L.) germplasm agro-morphological characterization based on descriptive, cluster and principal component analysis. Pakistan Journal of Botany, 47(SI), 255-264.

Jaradat, A. A. (2013). Perceptual distinctiveness in Native American maize (Zea mays L.) landraces has practical implications. Plant Genetic Resources: Characterization and Utilization, 11(3), 266-278. https://doi.org/ $10.1017 / \mathrm{S} 1479262113000063$

Jaradat, A. A., \& Goldstein, W. (2013). Diversity of maize kernels from a breeding program for protein quality: I. physical, biochemical, nutrient, and color traits. Crop Science, 53, 956-976. https://doi.org/10.2135/ cropsci2012.07.0437

Johnson, H. W., Robinson, H. F., \& Comstock, R. I. (1955). Estimates of genetic and environmental variability in soybeans. Agronomy Journal, 47, 314-318. https://doi.org/10.2134/agronj1955.00021962004700070009x

Kinfe, H., \& Tsehaye, Y. (2015). Studies of heritability, genetic parameters, correlation and path coefficient in elite maize hybrids. Academic Research Journal of Agricultural Science and Research, 3(10), 296-303. https://doi.org/10.14662/ARJASR2015.062.

Kwaga, Y. M. (2014). Correlation coefficients between grain yield and other characters of maize (Zea mays L.) grown at Mubi in Northern Savanna, Nigeria. International Journal of Farming and Allied Sciences, 3(2), 220-224.

Larson, W. E., \& Hanway, J. J. (1977). Corn production. In G. F. Sprague (Ed.), Corn and Corn Improvement (pp. 625-669). Madison, WI. ASA Inc.

Li, C. M., \& Yang, K. C. (1985). Studies on inheritance of quantitative characters for plant type in some inbred lines of maize. Scientia Agriculture Sinical, 19, 28-36.

McIntosh, M. S. (1983). Analysis of combined experiments. Agronomy Journal, 75, 53-155. https://doi.org/ 10.2134/agronj1983.00021962007500010041x

Motto, M., Hartings, H., Fracassetti, M., \& Consonni, G. (2011). Grain quality-related traits in maize: Gene identification and exploitation. Maydica, 56, 291-314.

Nataraj, V., Shahi, J. P., \& Vandana, D. (2014). Estimates of variability, heritability and genetic advance in certain inbreeds of maize (Zea mays L.). International Journal of Applied Biology and Pharmaceutical Technology, 5(1), 205-208.

Nuss, E. T., \& Tanumihardjo, S. A. (2010). Maize: A paramount staple crop in the context of global nutrition. Comprehensive Reviews in Food Science and Food Safety, 9, 417-436. https://doi.org/10.1111/j.1541-4337. 2010.00117.x

Rosales, A., Galicia, L., Oviedo, E., Islas, C., \& Palacios-Rojas, N. (2011). Near-infrared reflectance spectroscopy (NIRS) for protein, tryptophan, and lysine evaluation in quality protein maize (QPM) breeding programs. Journal of Agricultural and Food Chemistry, 59(20), 10781-10786. https://doi.org/10.1021/ jf201468x

Ross, A. J. (2002). Genetic analysis of ear length and correlated traits in maize (Ph.D. dissertation, Iowa State University).

Russell, I., \& Stewart, G. (2014). Whisky: Technology, Production and Marketing (2nd ed.). Amsterdam: Academic Press.

Scrob, S., Muste, S., Haş, I., Mureşan, C., Socaci, S., \& Fărcaş, A. (2014). The biochemical composition and correlation estimates for grain quality in maize. Journal of Agroalimentary Processes and Technologies, 20(2), 150-155.

Seebauer, J. R., Singletary, G. W., Krumpelman, P. M., Ruffo, M. L., \& Below, F. E. (2010). Relationship of 
source and sink in determining kernel composition of maize. Journal of Experimental Botany, 61, 511-519. https://doi.org/10.1093/jxb/erp324

Sharma, P., Punia, M. S., \& Kamboj, M. C. (2015). Estimates of heritability, heterosis and inbreeding depression for yield and quality traits in maize. Forage Research, 41(3), 139-146.

Steel, R. G. D., \& Torie, H. (1980). Principles and procedures of statistics (2nd ed.). Biometrical Approach, New York, McGraw-Hill.

Taller, J. M., \& Bernardo, R. (2004). Diverse adapted populations for improving northern maize inbreds. Crop Science, 44, 1444-1449. https://doi.org/10.2135/cropsci2004.1444

Wang, L., Wang, Q., Liu, H., Liu, L., \& Du, Y. (2013). Determining the contents of protein and amino acids in peanuts using near-infrared reflectance spectroscopy. Journal of the Science of Food and Agriculture, 93, 118-124. https://doi.org/10.1002/jsfa.5738

\section{Copyrights}

Copyright for this article is retained by the author(s), with first publication rights granted to the journal.

This is an open-access article distributed under the terms and conditions of the Creative Commons Attribution license (http://creativecommons.org/licenses/by/4.0/). 